\title{
The Fibers and Range of Reduction Graphs in Ciliates
}

\author{
Robert Brijder and Hendrik Jan Hoogeboom \\ Leiden Institute of Advanced Computer Science, Universiteit Leiden, \\ Niels Bohrweg 1, 2333 CA Leiden, The Netherlands, \\ rbrijder@liacs.nl
}

July 4, 2018

\begin{abstract}
The biological process of gene assembly has been modeled based on three types of string rewriting rules, called string pointer rules, defined on so-called legal strings. It has been shown that reduction graphs, graphs that are based on the notion of breakpoint graph in the theory of sorting by reversal, for legal strings provide valuable insights into the gene assembly process. We characterize which legal strings obtain the same reduction graph (up to isomorphism), and moreover we characterize which graphs are (isomorphic to) reduction graphs.

More formally, let $\mathcal{R}$ be the function which assigns to each legal string $u$ its reduction graph $\mathcal{R}_{u}$. We characterize the fiber $\mathcal{R}^{-1}\left(\mathcal{R}_{u}\right)$ (modulo graph isomorphism) for each reduction graph $\mathcal{R}_{u}$. In fact we show that $\mathcal{R}^{-1}\left(\mathcal{R}_{u}\right)$ is the 'orbit' of $u$ under two types of string rewriting rules, which are in a way dual to two of the three types of string pointer rules. We also characterize the range of $\mathcal{R}$ in terms of easy-to-check conditions on graphs.
\end{abstract}

\section{Introduction}

Ciliates form a large group of one-cellular organisms that are able to transform one nucleus, called the micronucleus, into an astonishing different one, called the macronucleus. This intricate DNA transformation process is called gene assem$b l y$. Each gene in the micronucleus, called micronuclear gene, is transformed to a gene in the macronucleus, called macronuclear gene. The string pointer reduction system models gene assembly based on three types of string rewriting rules, called string pointer rules, defined on so-called legal strings [6]. In this model, a micronuclear gene is represented by a legal string $u$, while its macronuclear gene (with its waste products) is represented by the reduction graph of $u$ [5, 4]. The reduction graph is based on the notion of breakpoint graph in the theory of sorting by reversal $8,1,10$. 1

This research was supported by the Netherlands Organization for Scientific Research (NWO) project 635.100.006 "VIEWS". 
In this paper we characterize which graphs are (isomorphic to) reduction graphs (cf. Theorem 24). Obviously, these graphs should have the 'look and feel' of reduction graphs. For instance, each vertex label should occur exactly four times, and the second type of edges connect vertices of the same label. Once these elementary properties are satisfied, reduction graphs are characterized as having a connected pointer-component graph - a graph which represents the distribution of the vertex labels over the connected components. The characterization corresponds to an efficient algorithm. In this way we obtain a restriction on the form of the macronuclear structures that can possibly occur. We also provide a characterization that determines, given two legal strings, whether or not they have the same reduction graph (cf. Theorem 34). This may allow one to determine which micronuclear genes obtain the same macronuclear structure. It turns out that two legal strings obtain the same reduction graph (up to isomorphism) exactly when they can be transformed into each other by two types of string rewriting rules, which surprisingly are in a sense dual to the string positive rules and the string double rules (two of the three types of string pointer rules).

The latter characterization has other uses as well. In a sense, the reduction graph allows for a complete characterization of applicability of string negative rules, the other type of string pointer rules, during the transformation process [5, 3, 2, 4. Moreover, it has been shown that the reduction graph does not retain much information about the applicability of the other two types of rules 3]. Therefore, the legal strings that obtain the same reduction graph are exactly the legal strings that have similar characteristics concerning the string negative rule.

To establish both main results, we augment the (abstract) reduction graph with a set of merge-legal edges. We will show that some "valid" sets of merge-legal edges for a reduction graph allows one to "go back" to a legal string corresponding to this (abstract) reduction graph. In this way the existence of such valid set determines which graphs are (isomorphic to) reduction graphs. The first main result shows that the existence of such valid set is computationally easy to verify. Moreover, the set of all sets of merge-legal edges can be transformed into each other by flip operations. These flip operations can be defined in terms of the above mentioned dual string pointer rules on legal strings. This will establish the other main result.

This paper is organized as follows. Section 2 fixes notation of basic mathematical notions. In Section 3 we recall the string pointer reduction system, in Section 4 we recall the reduction graph and the pointer-component graph, and in Section $[5$ we generalize the notion of reduction graph and give an extension through merge-legal edges. In Section 6 we provide a preliminary characterization that determines which graphs are (isomorphic to) reduction graphs. In the next three sections, we strengthen the result to allow for efficient algorithms: in Section 7 we define the flip operation on sets of merge-legal edges, in Section 8 we show that the effect of flip operation corresponds to merging or splitting of connected components, and in Section 9 we prove the first main result, cf. Theorem 24. In Sections 10 and 11 we prove the second main result, cf. Theorem 34 . We conclude this paper with a discussion. 


\section{Mathematical Notation and Terminology}

In this section we recall some basic notions concerning functions, strings, and graphs. We do this mainly to fix the basic notation and terminology.

The symmetric difference of sets $X$ and $Y,(X \backslash Y) \cup(Y \backslash X)$, is denoted by $X \oplus Y$. The symmetric difference of a finite family of sets $\left(X_{i}\right)_{i \in A}$ is denoted by $\bigoplus_{i \in A} X_{i}$. The composition of functions $f: X \rightarrow Y$ and $g: Y \rightarrow Z$ is the function $g f: X \rightarrow Z$ such that $(g f)(x)=g(f(x))$ for every $x \in X$. The restriction of $f$ to a subset $A$ of $X$ is denoted by $f \mid A$, . The range $f(X)$ of $f$ will be denoted by $\operatorname{rng}(f)$. We define for $y \in Y, f^{-1}(y)=\{x \in X \mid f(x)=y\}$. If $Y=X$, then $f$ is called self-inverse if $f^{2}$ is the identity function. We will use $\lambda$ to denote the empty string.

We now turn to graphs. A (undirected) graph is a tuple $G=(V, E)$, where $V$ is a finite set and $E \subseteq\{\{x, y\} \mid x, y \in V\}$. The elements of $V$ are the vertices of $G$ and the elements of $E$ are the edges of $G$. In this paper we allow $x=y$, and therefore edges can be of the form $\{x, x\}=\{x\}$ - an edge of this form should be seen as an edge connecting $x$ to $x$, i.e., a 'loop' for $x$. The restriction of $G$ to $E^{\prime} \subseteq E$, denoted by $\left.G\right|_{E^{\prime}}$, is $\left(V, E^{\prime}\right)$. The order $|V|$ of $G$ is denoted by $o(G)$.

A multigraph is a (undirected) graph $G=(V, E, \epsilon)$, where parallel edges are possible. Therefore, $E$ is a finite set of edges and $\epsilon: E \rightarrow\{\{x, y\} \mid x, y \in V\}$ is the endpoint mapping.

A coloured base $B$ is a 4-tuple $(V, f, s, t)$ such that $V$ is a finite set, $s, t \in V$, and $f: V \backslash\{s, t\} \rightarrow \Gamma$ for some $\Gamma$. The elements of $V,\{\{x, y\} \mid x, y \in V, x \neq y\}$, and $\Gamma$ are called vertices, edges, and vertex labels for $B$, respectively.

A $n$-edge coloured graph, $n \geq 1$, is a tuple $G=\left(V, E_{1}, E_{2}, \cdots, E_{n}, f, s, t\right)$ where $B=(V, f, s, t)$ is a coloured base and, for $i \in\{1, \ldots, n\}, E_{i}$ is a set of edges for $B$. We also denote $G$ by $B\left(E_{1}, E_{2}, \cdots, E_{n}\right)$. We define $\operatorname{dom}(G)=\operatorname{rng}(f)$.

The previously defined notions and notation for graphs carry over to multigraphs and $n$-edge coloured graphs. Isomorphisms between graphs are defined in the usual way: they are considered isomorphic when they are equal modulo the identity of the vertices. Thus, multigraphs $G=(V, E, \epsilon)$ and $G^{\prime}=\left(V^{\prime}, E, \epsilon^{\prime}\right)$ are isomorphic if there is a bijection $\alpha: V \rightarrow V^{\prime}$ such that $\alpha \epsilon=\epsilon^{\prime}$, or more precisely, for $e \in E, \epsilon(e)=\left\{v_{1}, v_{2}\right\}$ implies $\epsilon^{\prime}(e)=\left\{\alpha\left(v_{1}\right), \alpha\left(v_{2}\right)\right\}$. We assume the reader is familiar with the notions of cycle and connected component in a graph. A graph is called connected if it has exactly one connected component, and it is called acyclic when it does not contain cycles.

\section{String Pointer Reduction System}

The string pointer reduction system is the model of gene assembly that is used in this paper. In this section we give a concise description of this system, omitting examples and motivation. We refer to [7] for an in-depth description of this model including motivation and examples.

We fix $\kappa \geq 2$, and define the alphabet $\Delta=\{2,3, \ldots, \kappa\}$. For $D \subseteq \Delta$, we define $\bar{D}=\{\bar{a} \mid a \in D\}$ and $\Pi=\Delta \cup \bar{\Delta}$. The elements of $\Pi$ will be called pointers. We use the 'bar operator' to move from $\Delta$ to $\bar{\Delta}$ and back from $\bar{\Delta}$ to $\Delta$. Hence, 
for $p \in \Pi, \overline{\bar{p}}=p$. For a string $u=x_{1} x_{2} \cdots x_{n}$ with $x_{i} \in \Pi$, the inverse of $u$ is the string $\bar{u}=\bar{x}_{n} \bar{x}_{n-1} \cdots \bar{x}_{1}$. For $p \in \Pi$, we define $\mathbf{p}=\left\{\begin{array}{ll}p & \text { if } p \in \Delta \\ \bar{p} & \text { if } p \in \bar{\Delta}\end{array}\right.$, i.e., $\mathbf{p}$ is the 'unbarred' variant of $p$. The domain of a string $v \in \Pi^{*}$ is $\operatorname{dom}(v)=\{\mathbf{p} \mid$ $p$ occurs in $v\}$. A legal string is a string $u \in \Pi^{*}$ such that for each $p \in \Pi$ that occurs in $u, u$ contains exactly two occurrences from $\{p, \bar{p}\}$. For a pointer $p$ and a legal string $u$, if both $p$ and $\bar{p}$ occur in $u$ then we say that both $p$ and $\bar{p}$ are positive in $u$; if on the other hand only $p$ or only $\bar{p}$ occurs in $u$, then both $p$ and $\bar{p}$ are negative in $u$.

Let $u=x_{1} x_{2} \cdots x_{n}$ be a legal string with $x_{i} \in \Pi$ for $1 \leq i \leq n$. For a pointer $p \in \Pi$ such that $\left\{x_{i}, x_{j}\right\} \subseteq\{p, \bar{p}\}$ and $1 \leq i<j \leq n$, the $p$-interval of $u$ is the substring $x_{i} x_{i+1} \cdots x_{j}$. Two distinct pointers $p, q \in \Pi$ overlap in $u$ if both $\mathbf{q} \in \operatorname{dom}\left(I_{p}\right)$ and $\mathbf{p} \in \operatorname{dom}\left(I_{q}\right)$, where $I_{p}\left(I_{q}\right.$, resp.) is the $p$-interval ( $q$-interval, resp.) of $u$.

The string pointer reduction system consists of three types of reduction rules, called string pointer rules, operating on legal strings. In this paper we will not consider these rules directly, but rather study the reduction graph (which is recalled in the next section) that captures essential properties of the rewriting system. For completeness we list the rules. For all $p, q \in \Pi$ with $\mathbf{p} \neq \mathbf{q}$ :

- the string negative rule for $p$ is defined by $\operatorname{snr}_{p}\left(u_{1} p p u_{2}\right)=u_{1} u_{2}$,

- the string positive rule for $p$ is defined by $\mathbf{s p r}_{p}\left(u_{1} p u_{2} \bar{p} u_{3}\right)=u_{1} \bar{u}_{2} u_{3}$,

- the string double rule for $p, q$ is defined by $\mathbf{s d r}_{p, q}\left(u_{1} p u_{2} q u_{3} p u_{4} q u_{5}\right)=$ $u_{1} u_{4} u_{3} u_{2} u_{5}$,

where $u_{1}, u_{2}, \ldots, u_{5}$ are arbitrary (possibly empty) strings over $\Pi$.

We say that legal strings $u$ and $v$ are equivalent, denoted by $u \approx v$, if there is homomorphism $\varphi: \Pi^{*} \rightarrow \Pi^{*}$ with $\varphi(p) \in\{p, \bar{p}\}$ and $\varphi(\bar{p})=\overline{\varphi(p)}$ for all $p \in \Pi$ such that $\varphi(u)=v$.

\section{Example 1}

Legal strings $2 \overline{2} 33$ and $\overline{2} 233$ are equivalent, while $2 \overline{2} 33$ are $2 \overline{2} \overline{3} 3$ are not.

Note that $\approx$ is an equivalence relation. Equivalent legal strings are characterized by their 'unbarred version' and their set of positive pointers.

The domain of a reduction rule $\rho$, denoted by $\operatorname{dom}(\rho)$, is defined by $\operatorname{dom}\left(\mathbf{s n r}_{p}\right)=$ $\operatorname{dom}\left(\mathbf{s p r}_{p}\right)=\{\mathbf{p}\}$ and $\operatorname{dom}\left(\mathbf{s d r}_{p, q}\right)=\{\mathbf{p}, \mathbf{q}\}$ for $p, q \in \Pi$. For a composition $\varphi=\rho_{n} \cdots \rho_{2} \rho_{1}$ of reduction rules $\rho_{1}, \rho_{2}, \ldots, \rho_{n}$, the domain, denoted by $\operatorname{dom}(\varphi)$, is $\operatorname{dom}\left(\rho_{1}\right) \cup \operatorname{dom}\left(\rho_{2}\right) \cup \cdots \cup \operatorname{dom}\left(\rho_{n}\right)$.

A composition $\varphi$ of reduction rules is called a reduction. Let $u$ be a legal string. We say that $\varphi$ is a reduction of $u$, if $\varphi$ is a reduction and $\varphi$ is applicable to (defined on) $u$. A successful reduction $\varphi$ of $u$ is a reduction of $u$ such that $\varphi(u)=\lambda$. We then also say that $\varphi$ is successful for $u$. For every legal string there exists a successful reduction, which in general is not unique 7 . 


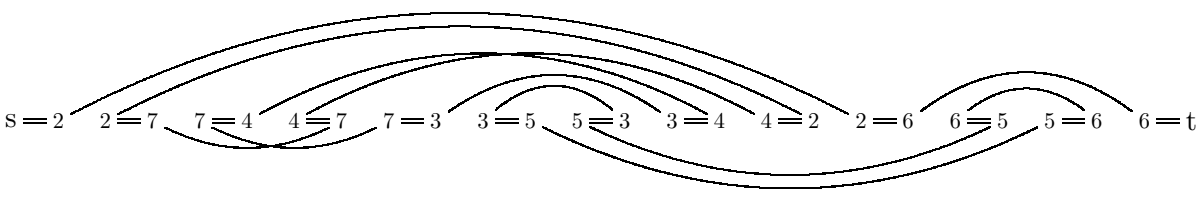

Figure 1: The reduction graph $\mathcal{R}_{u}$ of $u$ in Example 2,

\section{Reduction Graph}

We now recall the definition of reduction graph. This definition is equal to the one in [3], and is in slightly less general form compared to the one in [5]. We refer to [5, where it was introduced, for a motivation and for more examples and results. The notion of reduction graph uses the intuition from the notion of breakpoint graph (or reality-and-desire diagram) known from another branch of DNA processing theory called sorting by reversal, see e.g. [10] and [9. From a biological point of view, the reduction graph represents the macronuclear form of a gene given its micronuclear form. This micronuclear form of the gene is represented by a legal string, and therefore reduction graphs are defined on legal strings.

\section{Definition 1}

Let $u=p_{1} p_{2} \cdots p_{n}$ with $p_{1}, \ldots, p_{n} \in \Pi$ be a legal string. The reduction graph of $u$, denoted by $\mathcal{R}_{u}$, is a 2-edge coloured graph $\left(V, E_{1}, E_{2}, f, s, t\right)$, where

$$
\begin{gathered}
V=\left\{I_{1}, I_{2}, \ldots, I_{n}\right\} \cup\left\{I_{1}^{\prime}, I_{2}^{\prime}, \ldots, I_{n}^{\prime}\right\} \cup\{s, t\}, \\
E_{1}=\left\{e_{0}, e_{1}, \ldots, e_{n}\right\} \text { with } e_{i}=\left\{I_{i}^{\prime}, I_{i+1}\right\} \text { for } 1<i<n, e_{0}=\left\{s, I_{1}\right\}, e_{n}=\left\{I_{n}^{\prime}, t\right\}, \\
E_{2}=\left\{\left\{I_{i}^{\prime}, I_{j}\right\},\left\{I_{i}, I_{j}^{\prime}\right\} \mid i, j \in\{1,2, \ldots, n\} \text { with } i \neq j \text { and } p_{i}=p_{j}\right\} \cup \\
\left\{\left\{I_{i}, I_{j}\right\},\left\{I_{i}^{\prime}, I_{j}^{\prime}\right\} \mid i, j \in\{1,2, \ldots, n\} \text { and } p_{i}=\bar{p}_{j}\right\}, \text { and } \\
f\left(I_{i}\right)=f\left(I_{i}^{\prime}\right)=\mathbf{p}_{i} \text { for } 1 \leq i \leq n .
\end{gathered}
$$

The edges of $E_{1}$ are called the reality edges, and the edges of $E_{2}$ are called the desire edges. Notice that for each $p \in \operatorname{dom}(u)$, the reduction graph of $u$ has exactly two desire edges containing vertices labelled by $p$. It follows from the construction of the reduction graph that, given legal strings $u$ and $v, u \approx v$ implies that $\mathcal{R}_{u} \approx \mathcal{R}_{v}$.

In depictions of reduction graphs, we will represent the vertices (except for $s$ and $t$ ) by their labels, because the exact identity of the vertices is not essential for the problems considered in this paper. We will also depict reality edges as 'double edges' to distinguish them from the desire edges.

\section{Example 2}

The reduction graph of $u=2 \overline{7} 47353 \overline{4} 2656$ is depicted in Figure 1 Note how positive pointers are connected by crossing desire edges, while those for negative pointers are parallel. By rearranging the vertices we can depict the graph as shown in Figure 2 


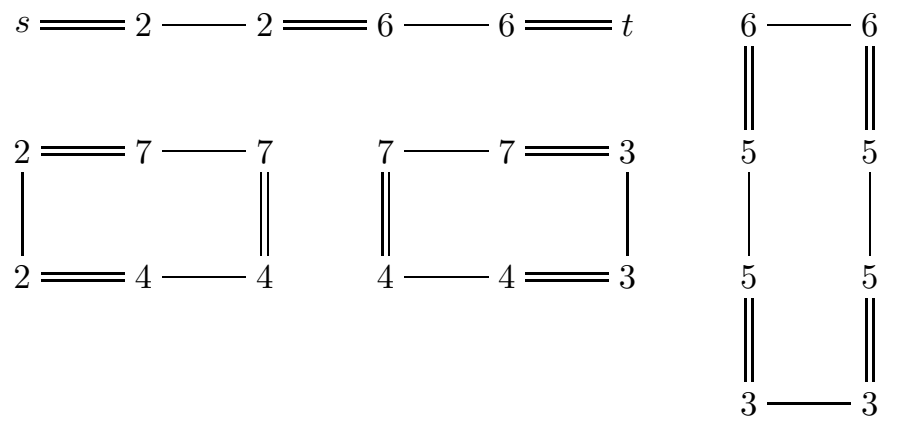

Figure 2: The reduction graph of Figure 1 obtained by rearranging the vertices.

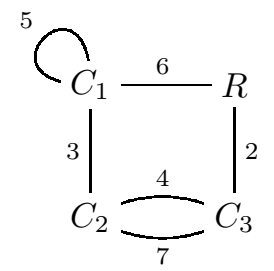

Figure 3: The pointer-component graph of the reduction graph from Figure 2.

Reality edges follow the linear order of the legal string, whereas desire edges connect positions in the string that will be joined when performing reduction rules, see [5].

We now recall the definition of pointer-component graph of a legal string, introduced in [3. Surprisingly however, this graph has different uses in this paper compared to its original uses in [3], where it is used to characterize which string negative rules are used in successful reductions of the legal string.

\section{Definition 2}

Let $u$ be a legal string. The pointer-component graph of $u$ (or of $\mathcal{R}_{u}$ ), denoted by $\mathcal{P} \mathcal{C}_{u}$, is a multigraph $(\zeta, E, \epsilon)$, where $\zeta$ is the set of connected components of $\mathcal{R}_{u}, E=\operatorname{dom}(u)$ and $\epsilon$ is, for $e \in E$, defined by $\epsilon(e)=\{C \in \zeta \mid C$ contains vertices labelled by $e\}$.

\section{Example 3}

The pointer-component graph of the reduction graph from Figure 2 is shown in Figure 3 .

\section{Abstract Reduction Graphs and Extensions}

In this section we generalize the notion of reduction graph as a starting point to consider which graphs are (isomorphic to) reduction graphs. Moreover, we extend the reduction graphs by a set of edges, called merge edges, such that, along with the reality edges, the linear structure of the legal string is preserved in the graph. 

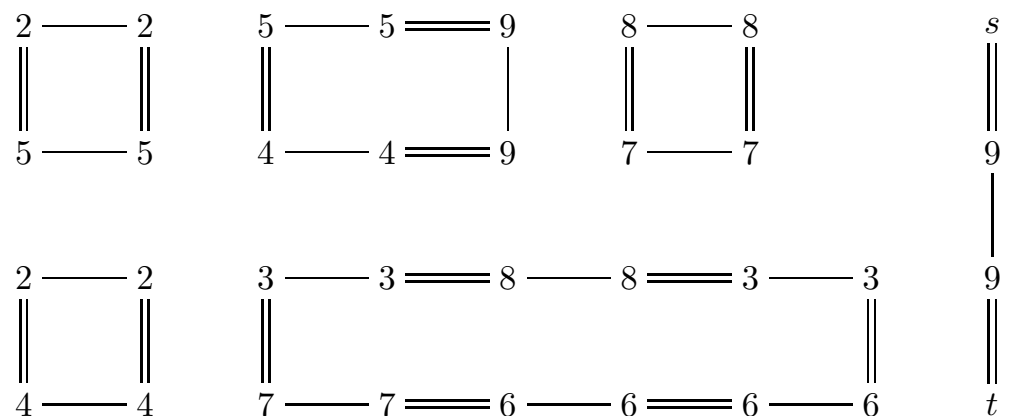

Figure 4: An abstract reduction graph.

We will now define a set of edges for a given coloured base which has features in common with desire edges of a reduction graph.

\section{Definition 3}

Let $B=(V, f, s, t)$ be a coloured base. We say that a set of edges $E$ for $B$ is desirable if

1. for all $\left\{v_{1}, v_{2}\right\} \in E, f\left(v_{1}\right)=f\left(v_{2}\right)$,

2. for each $v \in V \backslash\{s, t\}$ there is exactly one $e \in E$ such that $v \in e$.

We now generalize the concept of reduction graph.

\section{Definition 4}

A 2-edge coloured graph $B\left(E_{1}, E_{2}\right)$ with $B=(V, f, s, t)$ is called an abstract reduction graph if

1. $\operatorname{rng}(f) \subseteq \Delta$, and for each $p \in \operatorname{rng}(f),\left|f^{-1}(p)\right|=4$,

2. for each $v \in V$ there is exactly one $e \in E_{1}$ such that $v \in e$,

3. $E_{2}$ is desirable for $B$.

The set of all abstract reduction graphs is denoted by $\mathcal{G}$.

Clearly, if $G \approx \mathcal{R}_{u}$ for some $u$, then $G \in \mathcal{G}$. Therefore, for abstract reduction graphs $G=B\left(E_{1}, E_{2}\right)$, the edges in $E_{1}$ are called reality edges and the edges in $E_{2}$ are called desire edges. For graphical depictions of abstract reduction graphs we will use the same conventions as we have for reduction graphs. Thus, edges in $E_{1}$ will be depicted as "double edges", vertices are represented by their label, etc.

\section{Example 4}

The 2-edge coloured graph in Figure 4 is an abstract reduction graph.

Note that conditions (1) and (3) in the previous definition imply that for each $p \in \operatorname{rng}(f)$, there is a partition $\left\{e_{1}, e_{2}\right\}$ of $f^{-1}(p)$, denoted by $C_{G, p}$ or $C_{p}$ when $G$ is clear from the context, such that $e_{1}, e_{2} \in E_{2}$.

We now introduce an extension to reduction graphs such that the 'generic' linear order of the vertices $s, I_{1}, I_{1}^{\prime}, \ldots, I_{n}, I_{n}^{\prime}, t$ is retained, even when we consider the graphs up to isomorphism. 


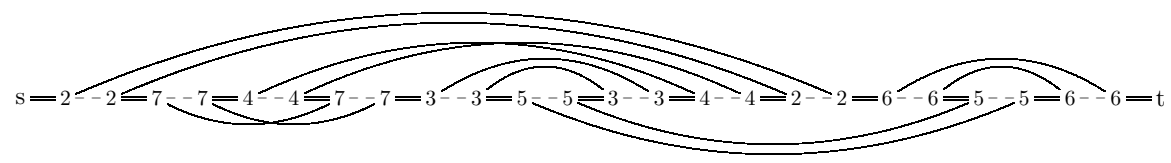

Figure 5: The extended reduction graph $\mathcal{E}_{u}$ of $u$ given in Example 2,

\section{Definition 5}

Let $u$ be a legal string. The extended reduction graph of $u$, denoted by $\mathcal{E}_{u}$, is a 3edge coloured graph $B\left(E_{1}, E_{2}, E_{3}\right)$, where $\mathcal{R}_{u}=B\left(E_{1}, E_{2}\right)$ and $E_{3}=\left\{\left\{I_{i}, I_{i}^{\prime}\right\} \mid\right.$ $1 \leq i \leq n\}$ with $n=|u|$.

The edges in $E_{3}$ are called the merge edges of $u$, denoted by $M_{u}$. In this way, the reality edges and the merge edges form a unique path which passes through the vertices in the generic linear order. This is illustrated in the next example. In figures merge edges will be depicted by "dashed edges".

\section{Example 5}

The extended reduction graph $\mathcal{E}_{u}$ of $u$ given in Example2 2 is shown in Figure 5 , cf. Figure 1,

\section{Remark}

The notion of merge edges for (extended) reduction graphs is more closely related to the notion of reality edges for breakpoint graphs in the theory of sortingby-reversal compared to the notion of reality edges for (extended) reduction graphs. Thus in a way it would be more natural to call the merge edges reality edges for (extended) reduction graphs, and the other way around. However, to avoid confusion with earlier work, we do not change this terminology.

We now generalize this extension of reduction graphs to abstract reduction graphs.

\section{Definition 6}

Let $G=B\left(E_{1}, E_{2}\right) \in \mathcal{G}$, and let $E$ be a set of edges for $B$. We say that $E$ is merge-legal for $G$ if $E$ is desirable for $B$, and $E_{2} \cap E=\varnothing$. We denote the set $\{E \mid E$ merge-legal for $G\}$ by $\omega_{G}$. The set of all $E \in \omega_{G}$ where $B\left(E_{1}, E\right)$ is connected is denoted by $\theta_{G}$.

For legal string $u$, we also denote $\omega_{\mathcal{R}_{u}}$ and $\theta_{\mathcal{R}_{u}}$ by $\omega_{u}$ and $\theta_{u}$, respectively.

Notice that $M_{u} \in \theta_{u} \subseteq \omega_{u}$. Therefore, merge-legal edges will also be depicted by "dashed edges".

\section{Example 6}

Let us consider the abstract reduction graph $G=B\left(E_{1}, E_{2}\right)$ of Figure 6. This graph is again depicted in Figure 7 including a merge-legal set $E$ for $G$. In this way Figure 7 depicts the 3 -edge coloured graph $B\left(E_{1}, E_{2}, E\right)$. Notice that $E \notin \theta_{G}$. In Figure 8, the abstract reduction graph is depicted with a merge-legal set in $\theta_{G}$.

We now define a natural abstraction of the notion of extended reduction graph. 

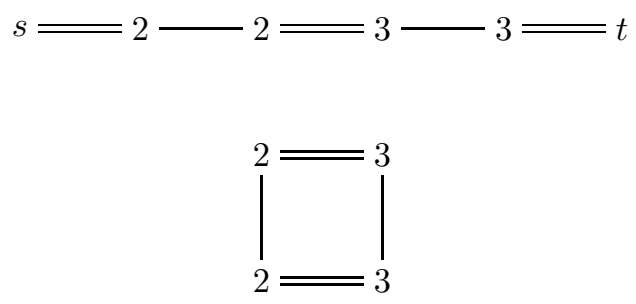

Figure 6: An abstract reduction graph.

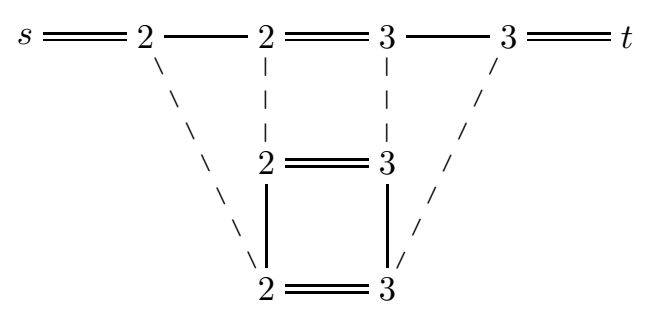

Figure 7: The abstract reduction graph of Figure 6 with a set of merge-legal edges.

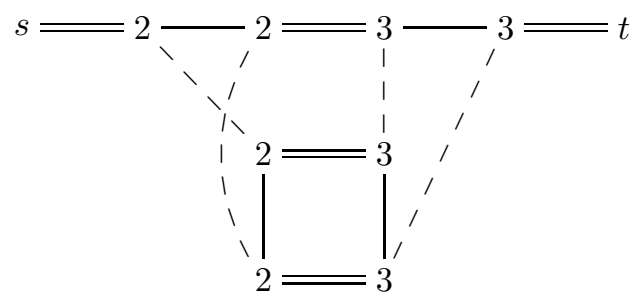

Figure 8: The abstract reduction graph of Figure 6 with another set of mergelegal edges. 


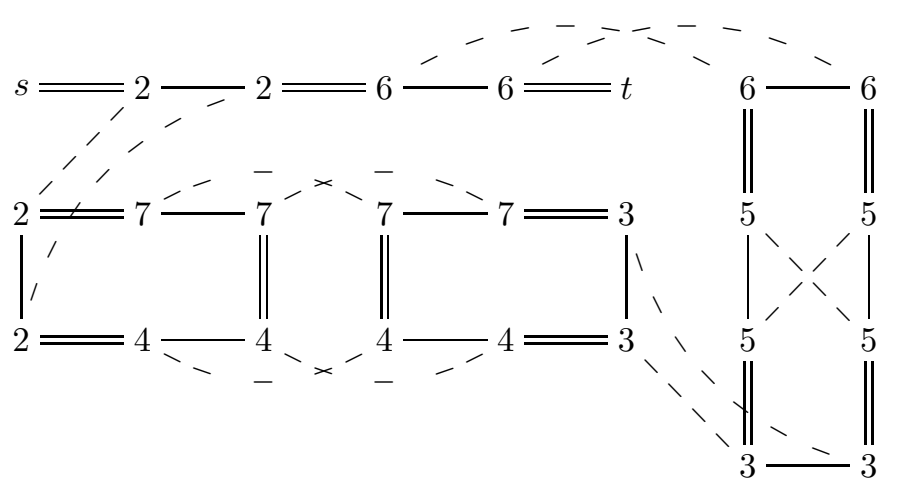

Figure 9: A extended abstract reduction graph obtained by augmenting the reduction graph of Figure 2 with merge edges.

\section{Definition 7}

Let $G=B\left(E_{1}, E_{2}\right) \in \mathcal{G}$ and $E \in \theta_{G}$. Then $G^{\prime}=B\left(E_{1}, E_{2}, E\right)$ is called a extended abstract reduction graph.

For each legal string $u, \mathcal{E}_{u}$ is an extended abstract reduction graph, since $M_{u} \in$ $\theta_{u}$. Therefore, the edges in $E$ (in the previous definition) are called the merge edges (of $G^{\prime}$ ). Since $E \in \theta_{G}, B\left(E_{1}, E\right)$ has the following form:

$$
s=\mathbf{p}_{1---} \mathbf{p}_{1}=\mathbf{p}_{2---\mathbf{p}_{2}=\cdots}=\mathbf{p}_{n---\mathbf{p}_{n}}=t
$$

Thus the property that reality and merge edges in an extended reduction graph induce a unique path from $s$ to $t$ that alternatingly passes through reality edges and merge edges is retained for extended abstract reduction graphs $G$ in general.

\section{Example 7}

If we consider the reduction graph $\mathcal{R}_{u}=B\left(E_{1}, E_{2}\right)$ of Example 2 shown in Figure 2 then, of course, $B\left(E_{1}, E_{2}, M_{u}\right)=\mathcal{E}_{u}$ shown in Figure 5 is a extended abstract reduction graph. In Figure 9 another extended reduction graph is shown - it is $\mathcal{R}_{u}$ augmented with a set of merge edges $E$ in $\theta_{u}$. It is easy to see that indeed $E \in \theta_{u}$ : simply notice that the path from $s$ to $t$ induced by the reality and merge edges will go through every vertex of the graph.

\section{$6 \quad$ Back to Legal Strings}

In this section we show that for extended abstract reduction graphs $G$ we can 'go back' in the sense that there are legal strings $u$ such that $G$ is isomorphic to $\mathcal{E}_{u}$. Moreover we show how to obtain the set $L_{G}$ of all legal strings that corresponds to $G$. We will show that the legal strings in $L_{G}$ are equivalent, and thus that extended reduction graphs retain all essential information of the legal strings. 


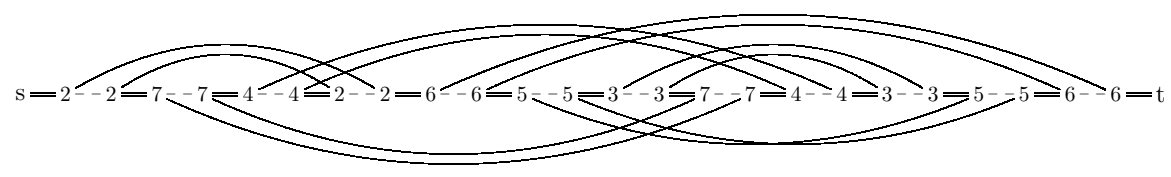

Figure 10: The extended abstract reduction graph $G$ given in Example 8

As extended abstract reduction graphs have a natural linear order of the vertices given by their reality edges and merge edges, we can infer whether or not desire edges 'cross' or not. Thereby providing a way to define negative and positive pointers for extended abstract reduction graphs.

\section{Definition 8}

Let $G=B\left(E_{1}, E_{2}, E_{3}\right)$ be an extended abstract reduction graph, let $G^{\prime}=$ $B\left(E_{1}, E_{2}\right)$, and let $\pi=\left(s, v_{1}, v_{1}^{\prime}, \cdots, v_{n}, v_{n}^{\prime}, t\right)$ be the path from $s$ to $t$ in $B\left(E_{1}, E_{3}\right)$. We say that $p \in \operatorname{dom}(G)$ is negative in $G$ iff $C_{G^{\prime}, p}=\left\{\left\{v_{i}, v_{j}^{\prime}\right\},\left\{v_{i}^{\prime}, v_{j}\right\}\right\}$ for some $i, j \in\{1, \ldots, n\}$ with $i \neq j$. Also, we say that $p \in \operatorname{dom}(G)$ is positive in $G$ if $p$ is not negative in $G$.

Clearly, $p \in \operatorname{dom}(G)$ is positive in $G$ iff $C_{G^{\prime}, p}=\left\{\left\{v_{i}, v_{j}\right\},\left\{v_{i}^{\prime}, v_{j}^{\prime}\right\}\right\}$ for some $i, j \in\{1, \ldots, n\}$ with $i \neq j$. It is easy to see that $p$ is negative in legal string $u$ iff $p$ is negative in $\mathcal{E}_{u}$.

The next definition defines a set of legal strings for each extended abstract reduction graph.

\section{Definition 9}

Let $G=B\left(E_{1}, E_{2}, E_{3}\right)$ be an extended abstract reduction graph, let $G^{\prime}=$ $B\left(E_{1}, E_{2}\right)$, and let $H=B\left(E_{1}, E_{3}\right)$ be as follows:

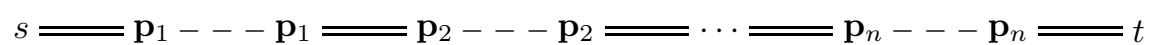

The legalization of $G$, denoted by $L_{G}$, is the set of legal strings $u=p_{1} p_{2} \cdots p_{n}$ with $p_{i} \in\left\{\mathbf{p}_{i}, \overline{\mathbf{p}}_{i}\right\}$ and $p_{i}$ is negative in $u$ iff $p_{i}$ is negative in $G$.

\section{Example 8}

Let us consider the extended abstract reduction graph $G$ of Figure 9. By rearranging the vertices we obtain Figure 10, From this figure it is clear that $v=2748826 \overline{5} 374356 \in L_{G}$.

It is easy to see that, for a legal string $u$, we have $u \in L_{\mathcal{E}_{u}}$.

Note that $L_{G}$, for extended abstract reduction graph $G$, is an non-empty equivalence class w.r.t. to the $\approx$ relation (for legal strings). Since the definition of $L_{G}$ does not depend on the exact identity of the vertices of $G$, we have, for extended abstract reduction graphs $G$ and $G^{\prime}, G \approx G^{\prime}$ implies $L_{G}=L_{G^{\prime}}$.

\section{Theorem 10}

1. Let $G$ and $G^{\prime}$ be extended abstract reduction graphs. Then $G \approx G^{\prime}$ iff $L_{G}=L_{G^{\prime}}$.

2. Let $u$ and $v$ be legal strings. Then $u \approx v$ iff $\mathcal{E}_{u} \approx \mathcal{E}_{v}$. 


\section{Proof}

We first consider statement 1 . We have already established the forward implication. We now prove the reverse implication. Let $G=B\left(E_{1}, E_{2}, E_{3}\right), G^{\prime}=$ $B^{\prime}\left(E_{1}^{\prime}, E_{2}^{\prime}, E_{3}^{\prime}\right)$, and $L_{G}=L_{G^{\prime}}$. By the definition of legalization, $B\left(E_{1}, E_{3}\right) \approx$ $B^{\prime}\left(E_{1}^{\prime}, E_{3}^{\prime}\right)$ and $p$ is negative in $G$ iff $p$ is negative in $G^{\prime}$ for $p \in \operatorname{dom}(G)=$ $\operatorname{dom}\left(G^{\prime}\right)$. Therefore, $G \approx G^{\prime}$.

We now consider statement 2 . We have $u \approx v$ iff $u, v \in L_{\mathcal{E}_{u}}=L_{\mathcal{E}_{v}}$ (since legalizations are equivalence classes of legal strings w.r.t $\approx$ ) iff $\mathcal{E}_{u} \approx \mathcal{E}_{v}$ (by the first statement).

Let $G$ be an extended abstract reduction graph, and take $u \in L_{G}$ (such a $u$ exists since $L_{G}$ is nonempty). Since $u \in L_{\mathcal{E}_{u}}$ and legalizations are equivalence classes, we have $L_{\mathcal{E}_{u}}=L_{G}$ and therefore $G \approx \mathcal{E}_{u}$. Thus every extended abstract reduction graph $G$ is isomorphic to an extended reduction graph. In fact, it is isomorphic to precisely those extended reduction graphs $\mathcal{E}_{u}$ with $u \in L_{G}$. Therefore, this $u$ is unique up to equivalence.

\section{Corollary 11}

Let $u$ and $v$ be legal strings. If $\mathcal{R}_{u} \approx \mathcal{R}_{v}$, then there is a $E \in \theta_{u}$ such that $\mathcal{E}_{v} \approx B\left(E_{1}, E_{2}, E\right)$ with $\mathcal{R}_{u}=B\left(E_{1}, E_{2}\right)$.

\section{Proof}

Since $\mathcal{R}_{u} \approx \mathcal{R}_{v}$, there is an set of edges $E$ for $\mathcal{R}_{u}$ such that $\mathcal{E}_{v} \approx B\left(E_{1}, E_{2}, E\right)$. Since $M_{v} \in \theta_{v}$, we have $E \in \theta_{u}$.

We end this section with a graph theoretical characterization of reduction graphs.

\section{Theorem 12}

Let $G$ be a 2-edge coloured graph. Then $G$ is isomorphic to a reduction graph iff $G \in \mathcal{G}$ and $\theta_{G} \neq \varnothing$.

\section{Proof}

Let $G \approx \mathcal{R}_{u}$ for some legal string $u$. Then clearly, $G \in \mathcal{G}$. Also, $M_{u} \in \theta_{u}$ and hence $\theta_{u} \neq \varnothing$. Therefore, $\theta_{G} \neq \varnothing$.

Let $E \in \theta_{G}$. Then $G^{\prime}=B\left(E_{1}, E_{2}, E\right)$ is an extended abstract reduction graph with $G=B\left(E_{1}, E_{2}\right)$. By the paragraph below Theorem 10, $G^{\prime} \approx \mathcal{E}_{u}$ for some legal string $u$ (take $u \in L_{G^{\prime}}$ ). Hence, $G \approx \mathcal{R}_{u}$.

\section{$7 \quad$ Flip Edges}

In this section and the next two we provide characterizations of the statement $\theta_{G} \neq \varnothing$. This allows, using Theorem 12, for a characterization that corresponds to an efficient algorithm that determines whether or not a given $G \in \mathcal{G}$ is isomorphic to a reduction graph. Moreover, it allows for an efficient algorithm that determines a legal string $u$ for which $G \approx \mathcal{R}_{u}$.

Let $G \in \mathcal{G}$. Then a merge-legal set for $G$ is easily obtained. For each $p \in \operatorname{dom}(G)$ with $C_{p}=\left\{\left\{v_{1}, v_{2}\right\},\left\{v_{3}, v_{4}\right\}\right\}$, a merge-legal set for $G$ must have either the edges $\left\{v_{1}, v_{3}\right\}$ and $\left\{v_{2}, v_{4}\right\}$ or the edges $\left\{v_{1}, v_{4}\right\}$ and $\left\{v_{2}, v_{3}\right\}$, see both sides in 

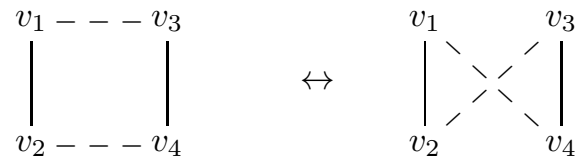

Figure 11: Flip operation for $p$. All vertices are labelled by $p$
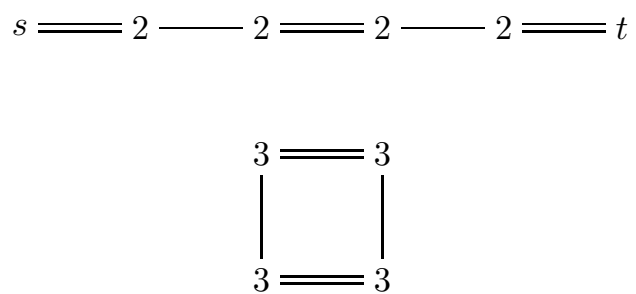

Figure 12: An abstract reduction graph $G$ for which $\theta_{G}=\varnothing$.

Figure 11, By assigning such edges for each $p \in \operatorname{dom}(G)$ we obtain a merge-legal set for $G$. Thus, $\omega_{G} \neq \varnothing$ for each $G \in \mathcal{G}$. Note that in particular, if $\operatorname{dom}(G)=\varnothing$, then $\omega_{G}=\{\varnothing\}$. However, $\theta_{G}$ can be empty as the next example will illustrate.

\section{Example 9}

It is easy to see that the abstract reduction graph $G$ of Figure 12 does not have a merge-legal set in $\theta_{G}$.

We now formally define a type of operation that in Figure 11 transforms the situation on the left-hand side to the situation on the right-hand side, and the other way around. Informally speaking it "flips" edges of merge-legal sets.

\section{Definition 13}

Let $G=B\left(E_{1}, E_{2}\right) \in \mathcal{G}$, let $f$ be the vertex labeling function of $G$, and let $p \in \operatorname{dom}(G)$. The flip operation for $p$ (w.r.t. $G$ ), denoted by $\operatorname{flip}_{G, p}$, is the function $\omega_{G} \rightarrow \omega_{G}$ defined by:

$$
\operatorname{flip}_{G, p}(E)=\left\{\left\{v_{1}, v_{2}\right\} \in E \mid f\left(v_{1}\right) \neq p \neq f\left(v_{2}\right)\right\} \cup\left\{e_{1}, e_{2}\right\},
$$

where $e_{1}$ and $e_{2}$ are the two edges with vertices labelled by $p$ such that $e_{1}, e_{2} \notin$ $E_{2} \cup E$.

When $G$ is clear from the context, we also denote flip $_{G, p}$ by $\operatorname{flip}_{p}$.

Since by Figure 11, there are exactly two edges $e_{1}$ and $e_{2}$ with vertices labelled by $p$ that are not parallel to both the edges in $E_{2} \cup E$, flip $p$ is well defined. It is now easy to see that indeed $\operatorname{flip}_{p}(E) \in \omega_{G}$ for $E \in \omega_{G}$.

\section{Example 10}

Let $G$ be the abstract reduction graph of Figure 6] If we apply flip f $_{G, 2}$ to the set of merge-legal edges depicted in Figure 7 , then we obtain the set of merge-legal edges depicted in Figure 8. 


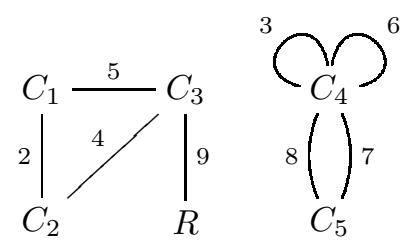

Figure 13: The pointer-component graph of the abstract reduction graph from Figure 4.

The next theorem follows directly from the previous definition and from the fact that Figure 11 contains the only possible ways in which edges in merge-legal sets for $G$ can be connected.

\section{Theorem 14}

Let $G \in \mathcal{G}$, and denote by $\mathcal{F}$ be the group generated by the flip operations w.r.t. $G$ under function composition. Then each element of $\mathcal{F}$ is self-inverse, thus $\mathcal{F}$ is Abelian, and $\mathcal{F}$ acts transitively on $\omega_{G}$.

Let $D=\left\{p_{1}, \ldots, p_{l}\right\} \subseteq \operatorname{dom}(G)$. Then we define flip $_{D}=$ flip $_{p_{l}} \cdots$ flip $_{p_{1}}$. Since $\mathcal{F}$ is Abelian, flip ${ }_{D}$ is well defined. Moreover, since each each element in $\mathcal{F}$ is selfinverse, $\mathcal{F}=\left\{\right.$ flip $\left._{D} \mid D \subseteq \operatorname{dom}(G)\right\}$. Also, if $D_{1}, D_{2} \subseteq \operatorname{dom}(G)$ and $D_{1} \neq D_{2}$, then $\operatorname{flip}_{D_{1}}(E) \neq \operatorname{flip}_{D_{2}}(E)$. Thus the following holds.

Theorem 15

Let $G \in \mathcal{G}$. Then there is a bijection $Q: 2^{\operatorname{dom}(G)} \rightarrow \mathcal{F}$ given by $Q(D)=\operatorname{flip}_{D}$. Moreover, for each $E \in \omega_{G}, \omega_{G}=\left\{\operatorname{flip}_{D}(E) \mid D \subseteq \operatorname{dom}(G)\right\}$.

\section{Merging and Splitting Connected Components}

Let $G=B\left(E_{1}, E_{2}\right)$ be an abstract reduction graph and let $E \in \omega_{G}$. In this section we consider the effect of the flip operation on the pointer-component graph defined on the abstract reduction graph $H=B\left(E_{1}, E\right)$. If we are able to obtain, using flip operations, a pointer-component graph consisting of one vertex, then $\theta_{G} \neq \varnothing$, and consequently by Theorem $12, G$ is isomorphic to a reduction graph.

However, first we need to define the notion of pointer-component graph for abstract reduction graphs in general. Fortunately, this generalization is trivial.

\section{Definition 16}

Let $G \in \mathcal{G}$. The pointer-component graph of $G$, denoted by $\mathcal{P C}$, is a multigraph $(\zeta, E, \epsilon)$, where $\zeta$ is the set of connected components of $G, E=\operatorname{dom}(G)$, and $\epsilon$ is, for $e \in E$, defined by $\epsilon(e)=\{C \in \zeta \mid C$ contains vertices labelled by $e\}$.

\section{Example 11}

The pointer-component graph of the graph from Figure 4 is shown in Figure 13.

Note that when $G=B\left(E_{1}, E_{2}\right) \in \mathcal{G}$ and $E \in \omega_{G}$, then $E$ is desirable for $B$. Hence, $H=B\left(E_{1}, E\right)$ is also an abstract reduction graph. Therefore, e.g., $\mathcal{P C}_{H}$ is defined. 
It is useful to distinguish the pointers that form loops in the pointer-component graph. Therefore, we define, for $G \in \mathcal{G}$, bridge $(G)=\{e \in E|| \epsilon(e) \mid=2\}$ where $\mathcal{P C}_{G}=(V, E, \epsilon)$. In [3], bridge $(G)$ is denoted as $\operatorname{snrdom}(G)$. However, this notation does not make sense for its uses in this paper.

\section{Example 12}

From Figure 13 it follows that bridge $(G)=\operatorname{dom}(G) \backslash\{3,6\}$ for the abstract reduction graph $G$ depicted in Figure 4 .

Merge rules have been used for multigraphs, and pointer-component graphs in particular in 3. The definition presented here is slightly different from the one in 3 - here the pointer $p$ on which the merge rule is applied remains present after the rule is applied.

\section{Definition 17}

For each edge $p$, the $p$-merge rule, denoted by merge ${ }_{p}$, is a rule applicable to (defined on) multigraphs $G=(V, E, \epsilon)$ with $p \in \operatorname{bridge}(G)$. It is defined by

$$
\operatorname{merge}_{p}(G)=\left(V^{\prime}, E, \epsilon^{\prime}\right),
$$

where $V^{\prime}=(V \backslash \epsilon(p)) \cup\left\{v^{\prime}\right\}$ with $v^{\prime} \notin V$, and $\epsilon^{\prime}(e)=\left\{h\left(v_{1}\right), h\left(v_{2}\right)\right\}$ iff $\epsilon(e)=$ $\left\{v_{1}, v_{2}\right\}$ where $h(v)=v^{\prime}$ if $v \in \epsilon(p)$, otherwise it is the identity.

It is easy to see that merge rules commute. We are now ready to state the following result which is similar to Theorem 27 in $[3]$.

\section{Theorem 18}

Let $G=B\left(E_{1}, E_{2}\right) \in \mathcal{G}$, let $E \in \omega_{G}$, let $H=B\left(E_{1}, E\right)$, and let, for $p \in \operatorname{dom}(G)$, $H_{p}=B\left(E_{1}, \operatorname{flip}_{p}(E)\right)$.

- If $p \in$ bridge $(H)$, then $\mathcal{P C} H_{p} \approx$ merge $_{p}\left(\mathcal{P C} \mathcal{C}_{H}\right)$ (and therefore $o\left(\mathcal{P C}_{H_{p}}\right)=o\left(\mathcal{P C}_{H}\right)-1$ ).

- If $p \in \operatorname{dom}(H) \backslash \operatorname{bridge}(H)$, then $o\left(\mathcal{P C}_{H}\right) \leq o\left(\mathcal{P C}_{H_{p}}\right) \leq o\left(\mathcal{P C}_{H}\right)+1$.

\section{Proof}

First let $p \in \operatorname{bridge}(H)$. Let $C_{H, p}=\left\{\left\{v_{1}, v_{2}\right\},\left\{v_{3}, v_{4}\right\}\right\}$. Then, $H$ has the following form, where each of the two edges in $C_{H, p}$ are from different connected components in $H$ and where, unlike our convention, we have depicted the vertices by their identity instead of their label:

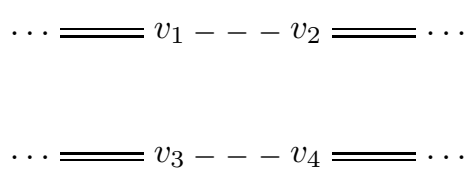

Now, either $\left\{\left\{v_{1}, v_{4}\right\},\left\{v_{2}, v_{3}\right\}\right\} \subseteq E_{2}$ or $\left\{\left\{v_{1}, v_{3}\right\},\left\{v_{2}, v_{4}\right\}\right\} \subseteq E_{2}$. Thus $H_{p}$ is of either

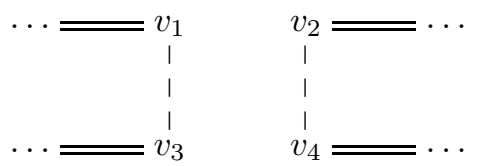




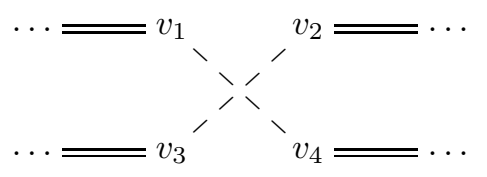

form, respectively. Thus in both cases, the two connected components are merged, and thus $\mathcal{P C}_{H_{p}}$ can be obtained (up to isomorphism) from $\mathcal{P C}_{H}$ by applying the merge ${ }_{p}$ operation.

Now let $p \in \operatorname{dom}(H) \backslash \operatorname{bridge}(H)$. Then the edges in $C_{H, p}$ belong to the same connected component. Thus $H$ has the following form

$$
\cdots=v_{1}--v_{2}=\cdots=v_{3}--v_{4}=\cdots
$$

where $C_{H, p}=\left\{\left\{v_{1}, v_{2}\right\},\left\{v_{3}, v_{4}\right\}\right\}$. Again, either $\left\{\left\{v_{1}, v_{4}\right\},\left\{v_{2}, v_{3}\right\}\right\} \subseteq E_{2}$ or $\left\{\left\{v_{1}, v_{3}\right\},\left\{v_{2}, v_{4}\right\}\right\} \subseteq E_{2}$. Thus $H_{p}$ is of either

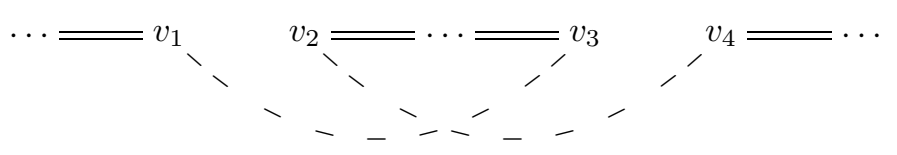

or

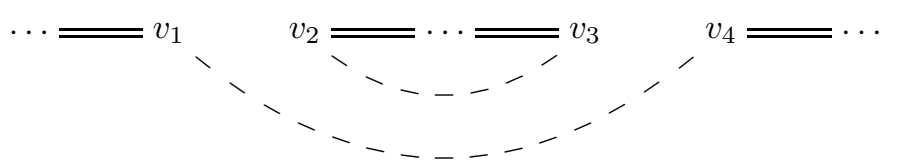

form, respectively. Thus, $H_{p}$ has either the same number of connected components of $H$ or exactly one more, respectively. Thus, $o\left(\mathcal{P C}_{H}\right) \leq o\left(\mathcal{P C}_{H_{p}}\right) \leq$ $o\left(\mathcal{P C}_{H}\right)+1$.

\section{Example 13}

Let $G=B\left(E_{1}, E_{2}\right) \in \mathcal{G}$ be as in Figure 6 . If we take $E \in \omega_{G}$ as in Figure 7 then $2 \in \operatorname{bridge}(H)$ with $H=B\left(E_{1}, E\right)$. Therefore, by Theorem 18 and the fact that $G$ has exactly two connected components, $H_{2}=B\left(E_{1}\right.$, $\left.\operatorname{flip}_{2}(E)\right)$ is a connected graph. Indeed, this is clear from Figure 8 (by ignoring the edges from $E_{2}$ ).

Informally, the next lemma shows that by applying flip operations, we can shrink a connected pointer-component graph to a single vertex. In this way, the underlying abstract reduction graph is a connected graph.

\section{Remark}

The next lemma appears to be similar to Lemma 29 in 3 . Although the flip operation (defined on graphs) and the rem operation (defined on strings) are quite distinct, they do have a similar effect on the pointer-component graph.

\section{Lemma 19}

Let $G=B\left(E_{1}, E_{2}\right) \in \mathcal{G}$, let $E \in \omega_{G}$, let $H=B\left(E_{1}, E\right)$, and let $D \subseteq \operatorname{dom}(G)=$ $\operatorname{dom}(H)$. Then $\left.\mathcal{P C}_{H}\right|_{D}$ is a tree iff $B\left(E_{1}, \operatorname{flip}_{D}(E)\right)$ and $H$ have 1 and $|D|+1$ connected components, respectively. 


\section{Proof}

Let $D=\left\{p_{1}, \ldots, p_{n}\right\}$. We first prove the forward implication. If $\left.\mathcal{P} \mathcal{C}_{H}\right|_{D}$ is a tree, then it has $|D|$ edges, and thus $|D|+1$ vertices. Therefore, $\mathcal{P C}_{H}$ has $|D|+1$ vertices, and consequently, $H$ has $|D|+1$ connected components. Since $\left.\mathcal{P C}_{H}\right|_{D}$ is acyclic, by Theorem 18 ,

$$
\mathcal{P C}_{B\left(E_{1}, \operatorname{fli}_{D}(E)\right)}=\mathcal{P C} \mathcal{C}_{B\left(E_{1},\left(\operatorname{flip}_{p_{n}} \cdots \operatorname{flip}_{p_{1}}\right)(E)\right)} \approx\left(\text { merge }_{p_{n}} \cdots \text { merge }_{p_{1}}\right)\left(\mathcal{P C}_{H}\right) .
$$

Now, applying $|D|$ merge operations on a graph with $|D|+1$ vertices, results in a graph containing exactly one vertex. Thus $B\left(E_{1}, \operatorname{flip}_{D}(E)\right)$ has one connected component.

We now prove the reverse implication. Moving from $H=B\left(E_{1}, E\right)$ to $B\left(E_{1}, f_{i j}(E)\right)$ reduces the number of connected components in $|D|$ steps from $|D|+1$ to 1 . By Theorem 18, each flip operation of flip $_{D}$ corresponds to a merge operation. Therefore (merge $p_{p_{n}} \cdots$ merge $_{p_{1}}$ ) is applicable to $\mathcal{P} \mathcal{C}_{H}$. Consequently, $\left.\mathcal{P C}_{H}\right|_{D}$ is acyclic. Since this graph has $|D|+1$ vertices, $\left.\mathcal{P C}_{H}\right|_{D}$ is a tree.

\section{Connectedness of Pointer-Component Graph}

In this section we use the results of the previous two sections to prove our first main result, cf. Theorem 24, which strengthens Theorem 12 by replacing the requirement $\theta_{G} \neq \varnothing$ by a simple test on $\mathcal{P C}_{G}$. We now characterize the connectedness of $\mathcal{P C}_{G}$.

\section{Definition 20}

Let $B=(V, f, s, t)$ be a coloured base. We say that a set of edges $E$ for $B$ is wellcoloured (for $B$ ) if for each partition $\rho=\left(V_{1}, V_{2}\right)$ of $V$ with $f\left(V_{1}\right) \cap f\left(V_{2}\right)=\varnothing$, there is an edge $\left\{v_{1}, v_{2}\right\} \in E$ with $v_{1} \in V_{1}$ and $v_{2} \in V_{2}$.

We call $G=B\left(E_{1}, E_{2}\right) \in \mathcal{G}$ well-coloured if $E_{1}$ is well-coloured for $B$.

\section{Lemma 21}

Let $G \in \mathcal{G}$. Then $\mathcal{P C}_{G}$ is a connected graph iff $G$ is well-coloured.

\section{Proof}

Let $G=B\left(E_{1}, E_{2}\right)$ with $B=(V, f, s, t)$. We first prove the forward implication. Let $G$ be not well-coloured. Then there is a partition $\rho=\left(V_{1}, V_{2}\right)$ of $V$ with $f\left(V_{1}\right) \cap f\left(V_{2}\right)=\varnothing$ such that for each $e \in E_{1}$, either $e \subseteq V_{1}$ or $e \subseteq V_{2}$. Since for each $\left\{v_{1}, v_{2}\right\} \in E_{2}$ we have $f\left(v_{1}\right)=f\left(v_{2}\right)$, we have either $\left\{v_{1}, v_{2}\right\} \subseteq V_{1}$ or $\left\{v_{1}, v_{2}\right\} \subseteq V_{2}$. Therefore $V_{1}$ and $V_{2}$ induce two non-empty sets of connected components which have no vertex label in common. Therefore, $\mathcal{P C}_{G}$ is not a connected graph.

We now prove the reverse implication. Assume that $\mathcal{P C}_{G}=(\zeta, E, \epsilon)$ is not a connected graph. Then, by the definition of pointer-component graph, there is a partition $\left(C_{1}, C_{2}\right)$ of $\zeta$ such that $C_{1}$ and $C_{2}$ have no vertex label in common. Let $V_{i}$ be the set of vertices of the connected components in $C_{i}(i \in\{1,2\})$. Then for partition $\rho=\left(V_{1}, V_{2}\right)$ of $V$ we have $f\left(V_{1}\right) \cap f\left(V_{2}\right)=\varnothing$ and for each $e \in E_{1} \cup E_{2}$, either $e \subseteq V_{1}$ or $e \subseteq V_{2}$. Therefore $G$ is not well-coloured. 
Clearly, if $G=B\left(E_{1}, E_{2}\right) \in \mathcal{G}$ is well-coloured and $E$ is desirable for $B$ (e.g., one could take $\left.E \in \omega_{G}\right)$, then $H=B\left(E_{1}, E\right) \in \mathcal{G}$ and $H$ is well-coloured. Therefore, by Lemma 21, $\mathcal{P C}_{G}$ is a connected graph iff $\mathcal{P C}$ is a connected graph.

By Theorem 12 the next result is essential to efficiently determine which abstract reduction graphs are isomorphic to reduction graphs.

Theorem 22

Let $G \in \mathcal{G}$. Then $\mathcal{P C}_{G}$ is a connected graph iff $\theta_{G} \neq \varnothing$.

Proof

Let $G=B\left(E_{1}, E_{2}\right)$. We first prove the forward implication. Let $\mathcal{P C}_{G}$ be a connected graph and let $E \in \omega_{G}$. Then $\mathcal{P C}_{H}$ with $H=B\left(E_{1}, E\right)$ is a con-

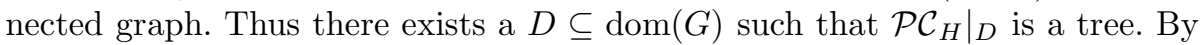
Lemma 19, $B\left(E_{1}\right.$, flip $\left._{D}(E)\right)$ is a connected graph, and consequently flip ${ }_{D}(E) \in$ $\theta_{G}$.

We now prove the reverse implication. Let $E \in \theta_{G}$. Thus, $H=B\left(E_{1}, E\right)$ is a connected graph, and hence $\mathcal{P C}_{H}$ is a connected graph. Therefore, $\mathcal{P} \mathcal{C}_{G}$ is also a connected graph.

We can summarize the last two results as follows.

\section{Corollary 23}

Let $G \in \mathcal{G}$. Then the following conditions are equivalent:

1. $G$ is well-coloured,

2. $\mathcal{P C}_{G}$ is a connected graph, and

3. $\theta_{G} \neq \varnothing$.

\section{Example 14}

By Figure 3 and Corollary 23, for (abstract) reduction graph $G_{1}$ in Figure 2 we have $\theta_{G_{1}} \neq \varnothing$. On the other hand, by Figure 13 and Corollary 23, for abstract reduction graph $G_{2}$ in Figure 4 we have $\theta_{G_{2}}=\varnothing$.

By Corollary 23 and Theorem 12 we obtain the first main result of this paper. It shows that one needs to check only a few computationally easy conditions to determine whether or not a 2-edge coloured graph is (isomorphic to) a reduction graph. Surprisingly, the 'high-level' notion of pointer-component graph is crucial in this characterization.

\section{Theorem 24}

Let $G$ be a 2-edge coloured graph. Then $G$ isomorphic to a reduction graph iff $G \in \mathcal{G}$ and $\mathcal{P C}_{G}$ is a connected graph.

Note that in the previous theorem we can equally well replace " $\mathcal{P C}$ is a connected graph" by one of the other equivalent conditions in Corollary 23 ,

In Theorem 21 in $[3]$ it is shown that the pointer-component graph of each reduction graph is a connected graph. We did not use that result here - in fact it is now a direct consequence of Theorem 24.

Not only is it computationally efficient to determine whether or not a 2-edge coloured graph $G$ is isomorphic to a reduction graph, but, when this is the case, then it is also computationally easy to determine a legal string $u$ for which $G \approx \mathcal{R}_{u}$. Indeed, we can determine such a $u$ from $G=B\left(E_{1}, E_{2}\right)$ as follows: 
1. Determine a $E \in \omega_{G}$. As we have mentioned before, such a $E$ is easily obtained.

2. Compute $\mathcal{P C}_{H}$ with $H=B\left(E_{1}, E\right)$, and determine a set of edges $D$ such that $\left.\mathcal{P} \mathcal{C}_{H}\right|_{D}$ is a tree.

3. Compute $G^{\prime}=B\left(E_{1}, E_{2}, \operatorname{flip}_{D}(E)\right)$, and determine a $u \in L_{G^{\prime}}$.

As a consequence, pointer-component graphs of legal strings can, surprisingly, take all imaginable forms.

\section{Corollary 25}

Every connected multigraph $G=(V, E, \epsilon)$ with $E \subseteq \Delta$ is isomorphic to a pointer-component graph of a legal string.

\section{Flip and the Underlying Legal String}

We now move to the second part of this paper, where we characterize the fibers $\mathcal{R}^{-1}\left(\mathcal{R}_{u}\right)$ modulo graph isomorphism. First we consider the effect of flip operations on the set of merge edges.

\section{Lemma 26}

Let $u$ be a legal string and let $p \in \operatorname{dom}(u)$. If $p$ is negative in $u$, then $\operatorname{flip}_{p}\left(M_{u}\right) \in$ $\theta_{u}$. If $p$ is positive in $u$, then $\operatorname{flip}_{p}\left(M_{u}\right) \notin \theta_{u}$. In other words, $\operatorname{flip}_{p}\left(M_{u}\right) \in \theta_{u}$ iff $p$ is negative in $u$.

\section{Proof}

Let $\mathcal{R}_{u}=B\left(E_{1}, E_{2}\right)$. By the definition of $\operatorname{flip}_{p}$, $\operatorname{flip}_{p}\left(M_{u}\right) \in \omega_{u}$. It suffices to prove that $G=B\left(E_{1}, f l i p_{p}\left(M_{u}\right)\right)$ is a connected graph when $p$ is negative in $u$ and not a connected graph when $p$ is positive in $u$. Graph $B\left(E_{1}, M_{u}\right)$ has the following form:

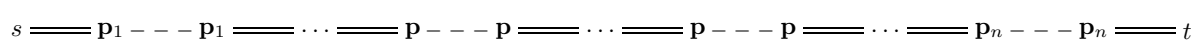

Now if $p$ is negative in $u$, then $G$ has the following form:

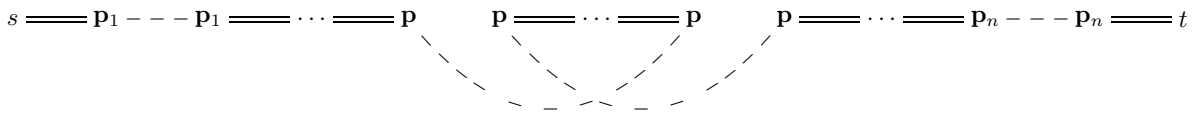

Thus in this case $G$ is connected.

If $p$ is positive in $u$, then $G$ has the following form:

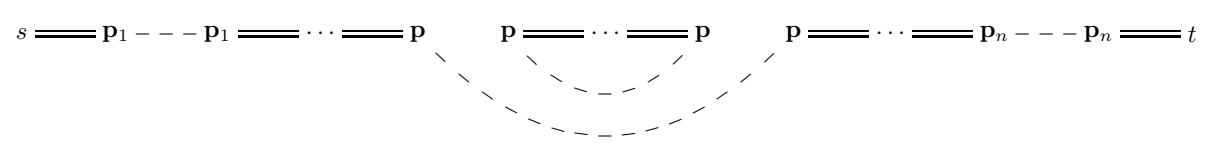

Thus in this case $G$ is not connected.

\section{Lemma 27}

Let $u$ be a legal string and let $p, q \in \operatorname{dom}(u)$. If $p$ and $q$ are overlapping in $u$ and not both negative in $u$, then $\operatorname{flip}_{\{p, q\}}\left(M_{u}\right) \in \theta_{u}$. 


\section{Proof}

Let $\mathcal{R}_{u}=B\left(E_{1}, E_{2}\right)$. Then $B\left(E_{1}, M_{u}\right)$ has the following form (we can assume without loss of generality that $p$ appears before $q$ in the path from $s$ to $t$ ):

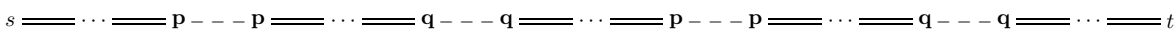

Assume that $p$ is positive in $u$ - the other case ( $q$ is positive in $u$ ) is proved similarly. By the proof of Lemma 26 it follows that $B\left(E_{1}, f_{i p}\left(M_{u}\right)\right)$ has the following form:

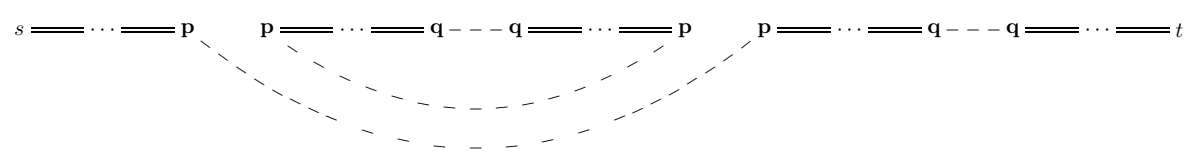

Therefore, $q \in \operatorname{bridge}\left(B\left(E_{1}\right.\right.$, flip $\left.\left._{p}\left(M_{u}\right)\right)\right)$. By Theorem 18 , the pointer-component graph of $B\left(E_{1}, \operatorname{flip}_{\{p, q\}}\left(M_{u}\right)\right)$ has only one vertex. Consequently, $B\left(E_{1}\right.$, flip $\left.\operatorname{lp}_{\{q\}}\left(M_{u}\right)\right)$

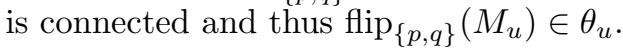

\section{Lemma 28}

Let $u$ be a legal string, and let $D \subseteq \operatorname{dom}(u)$ be nonempty. If $\operatorname{flip}_{D}\left(M_{u}\right) \in \theta_{u}$, then either there is a $p \in D$ negative in $u$ or there are $p, q \in D$ positive and overlapping in $u$.

\section{Proof}

Let $\mathcal{E}_{u}=B\left(E_{1}, E_{2}, M_{u}\right)$ and let $\operatorname{flip}_{D}\left(M_{u}\right) \in \theta_{u}$. Then $B\left(E_{1}, \operatorname{flip}_{D}\left(M_{u}\right)\right)$ is a connected graph. Assume to the contrary that all elements in $D$ are positive and pairwise non-overlapping in $u$. Then there is a $p \in D$ such that the domain of the $p$-interval does not contain an element in $D \backslash\{p\}$. By the proof of Lemma 26 $B\left(E_{1}\right.$, flip $\left._{p}\left(M_{u}\right)\right)$ consist of two connected components, one of which does not have vertices labelled by elements in $D \backslash\{p\}$. Therefore $B\left(E_{1}, f l i p_{D}\left(M_{u}\right)\right)$ also contains this connected component, and thus $B\left(E_{1}\right.$, flip $\left.{ }_{D}\left(M_{u}\right)\right)$ has more than one connected component - a contradiction.

By the previous lemmata, we have the following result.

\section{Theorem 29}

Let $u$ be a legal string, and let $D \subseteq \operatorname{dom}(u)$ be nonempty. If $\operatorname{flip}_{D}\left(M_{u}\right) \in \theta_{u}$, then either there is a $p \in D$ negative in $u$ with $\operatorname{flip}_{p}\left(M_{u}\right) \in \theta_{u}$ or there are $p, q \in D$ positive and overlapping in $u$ with $\operatorname{flip}_{\{p, q\}}\left(M_{u}\right) \in \theta_{u}$.

\section{Dual String Rules}

We now define the dual string rules. These rules will be used to characterize the effect of flip operations on the underlying legal string. For all $p, q \in \Pi$ with $\mathbf{p} \neq \mathbf{q}$ we define

- the dual string positive rule for $p$ is defined by $\mathbf{d s p r}_{p}\left(u_{1} p u_{2} p u_{3}\right)=u_{1} p \bar{u}_{2} p u_{3}$,

- the dual string double rule for $p, q$ is defined by $\mathbf{d s d r}_{p, q}\left(u_{1} p u_{2} q u_{3} \bar{p} u_{4} \bar{q} u_{5}\right)=$ $u_{1} p u_{4} q u_{3} \bar{p} u_{2} \bar{q} u_{5}$, 
where $u_{1}, u_{2}, \ldots, u_{5}$ are arbitrary (possibly empty) strings over $\Pi$. Notice that the dual string rules are self-inverse. Also notice the strong similarities between dspr and spr, and between dsdr and sdr. Both $\mathbf{d s p r}_{p}$ and $\mathbf{s p r}_{p}$ invert the substring between the two occurrences of $p$ or $\bar{p}$. However, $\mathbf{d s p r}_{p}$ is applicable when $p$ is negative, while $\mathbf{s p r}_{p}$ is applicable when $p$ is positive. Also, $\mathbf{s p r}_{p}$ removes the occurrences of $p$ and $\bar{p}$, while dspr does not. A similar comparison can be made between $\mathbf{d s d r}$ and sdr.

The domain of (sequences of) dual string rules is defined similarly as for string rules. Thus, e.g., $\operatorname{dom}\left(\mathbf{d s d r}_{p, q}\right)=\{p, q\}$.

\section{Definition 30}

Let $u$ and $v$ be legal strings. We say that $u$ and $v$ are dual, denoted by $\approx_{d}$ if there is a (possibly empty) sequence $\varphi$ of dual string rules applicable to $u$ such that $\varphi(u) \approx v$.

Notice that $\approx_{d}$ is an equivalence relation. Clearly, $\approx_{d}$ is reflexive. It is symmetrical since dual string rules are self-inverse, and it is transitive by function composition: if $\varphi_{1}(u) \approx v$ and $\varphi_{2}(v) \approx w$, then $\left(\varphi_{2} \varphi_{1}\right)(u) \approx w$.

Since $\mathbf{d s p r}_{p}$ is applicable when $p$ is negative in $u$ and $\mathbf{d s d r}_{p, q}$ is applicable when $p$ and $q$ are positive and overlapping, the following result is a direct corollary to Lemma 28.

\section{Corollary 31}

Let $u$ be a legal string, and let $D \subseteq \operatorname{dom}(u)$ be nonempty. If $\operatorname{flip}_{D}\left(M_{u}\right) \in \theta_{u}$, then there is a dual string rule $\rho$ with $\operatorname{dom}(\rho) \subseteq D$ applicable to $u$.

Let $\varphi=\rho_{n} \cdots \rho_{1}$ with each $\rho_{i}$ (for $1 \leq i \leq n$ ) a dual string rule. We define $\operatorname{odom}(\varphi)=\bigoplus_{1 \leq i \leq n} \operatorname{dom}\left(\rho_{i}\right)$. Thus, $\operatorname{odom}(\varphi) \subseteq \operatorname{dom}(\varphi)$. We call $\varphi$ reduced if $\operatorname{dom}\left(\rho_{i}\right) \cap \operatorname{dom}\left(\rho_{j}\right)=\varnothing$ for all $1 \leq i<j \leq n$. Note that if $\varphi$ is reduced, then $\operatorname{dom}(\varphi)=\operatorname{odom}(\varphi)$.

Let $G=B\left(E_{1}, E_{2}, E_{3}\right)$ be an extended abstract reduction graph, and let $D \subseteq$ $\operatorname{dom}(G)$. Then we define $\operatorname{flip}_{D}(G)=B\left(E_{1}, E_{2}, \operatorname{flip}_{G^{\prime}, D}\left(E_{3}\right)\right)$, where $G^{\prime}=B\left(E_{1}, E_{2}\right)$.

\section{Lemma 32}

Let $u$ be a legal string, and let $\varphi$ be a sequence of dual string rules applicable to $u$. Then $\mathcal{E}_{\varphi(u)} \approx \operatorname{flip}_{D}\left(\mathcal{E}_{u}\right)$ with $D=\operatorname{odom}(\varphi)$. Consequently, $\mathcal{R}_{\varphi(u)} \approx \mathcal{R}_{u}$.

\section{Proof}

It suffices to prove the result for the case $\varphi=\mathbf{d s p r}_{p}$ with $p \in \Pi$ and for the case $\varphi=\mathbf{d s d r}_{p, q}$ with $p, q \in \Pi$. We first prove the case where $\varphi=\mathbf{d s p r}_{p}$ for some $p \in \Pi$ is applicable to $u$. Then by the second figure in the proof of Lemma 26 we see that the inversion of the substring between the two occurrences of $p$ in $u$ accomplished by $\varphi$ faithfully simulates the corresponding effect of flip ${ }_{p}$ on $\mathcal{E}_{u}$. We only need to verify that $p$ is negative in $\operatorname{flip}_{p}\left(\mathcal{E}_{u}\right)$. To do this, we depict $\mathcal{E}_{u}$ such that the vertices are represented by their identity instead of their label:

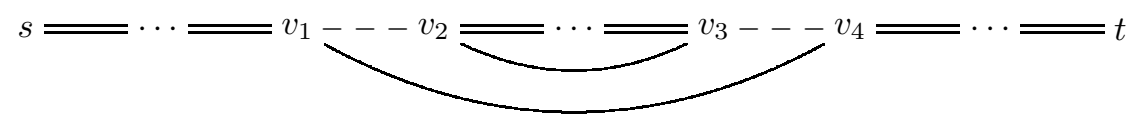


where the vertices $v_{i}, i \in\{1,2,3,4\}$, are labelled by $\mathbf{p}$. Then $\operatorname{flip}_{p}\left(\mathcal{E}_{u}\right)$ is

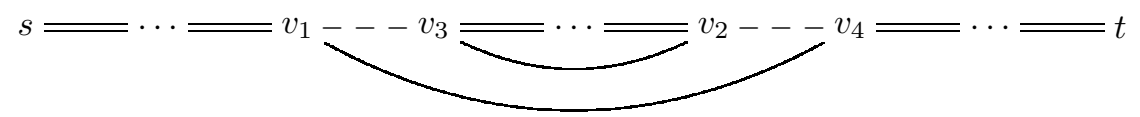

Therefore $p$ is indeed negative in $\operatorname{flip}_{p}\left(\mathcal{E}_{u}\right)$, and consequently $\mathcal{E}_{\varphi(u)} \approx \operatorname{flip}_{p}\left(\mathcal{E}_{u}\right)$. We now prove the case where $\varphi=\mathbf{d s d r}_{p, q}$ with $p, q \in \Pi$. Let $\mathcal{E}_{u}=B\left(E_{1}, E_{2}, E_{3}\right)$, then $\mathcal{E}_{u}$ has the following form

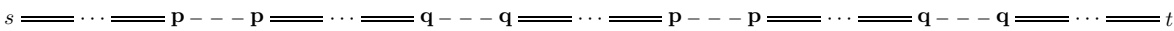

where we omitted the edges in $E_{2}$. Since $p$ and $q$ are positive in $u$, flip $\operatorname{sp}_{\{p, q\}}\left(\mathcal{E}_{u}\right)$ has the following form:

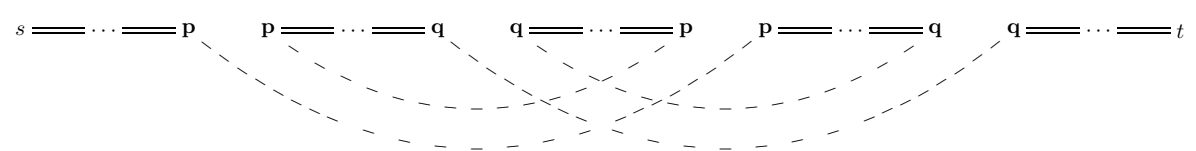

where we again omitted the edges in $E_{2}$. Thus, we see that interchanging the substring in $u$ between $p$ and $q$ and the substring in $u$ between $\bar{p}$ and $\bar{q}$ accomplished by $\varphi$ faithfully simulates the corresponding effect of flip $p_{p, q}$ on $\mathcal{E}_{u}$. We only need to verify that both $p$ and $q$ are positive in $\operatorname{flip}_{p, q}\left(\mathcal{E}_{u}\right)$. To do this, we depict $\mathcal{E}_{u}$ such that the vertices are represented by their identity instead of their label:

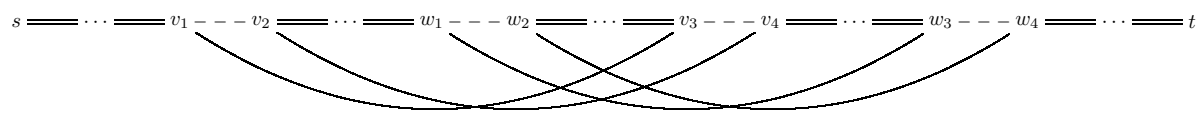

where the vertices $v_{i}$ and $w_{i}, i \in\{1,2,3,4\}$, are labelled by $\mathbf{p}$ and $\mathbf{q}$, respectively. Then $\operatorname{flip}_{p, q}\left(\mathcal{E}_{u}\right)$ is

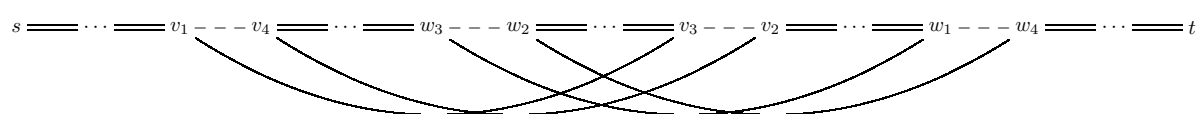

Therefore both $p$ and $q$ are indeed positive in $\operatorname{flip}_{p, q}\left(\mathcal{E}_{u}\right)$, and consequently $\mathcal{E}_{\varphi(u)} \approx \operatorname{flip}_{p, q}\left(\mathcal{E}_{u}\right)$.

Thus, if $\varphi_{1}$ and $\varphi_{2}$ are sequences of dual string rules applicable to a legal string $u$ with $\operatorname{odom}\left(\varphi_{1}\right)=\operatorname{odom}\left(\varphi_{2}\right)$, then $\mathcal{E}_{\varphi_{1}(u)} \approx \mathcal{E}_{\varphi_{2}(u)}$ and thus $\varphi_{1}(u) \approx \varphi_{2}(u)$.

\section{Lemma 33}

Let $u$ be a legal string, and let $D \subseteq \operatorname{dom}(u)$. There is a reduced sequence $\varphi$ of dual string rules applicable to $u$ such that $\operatorname{dom}(\varphi)=D$ iff $\operatorname{flip}_{D}\left(M_{u}\right) \in \theta_{u}$.

\section{Proof}

The forward implication follows directly from Lemma 32 . We now prove the reverse implication. If $D=\varnothing$, we have nothing to prove. Let $D \neq \varnothing$. By Corollary 31, there is a dual string rule $\rho_{1}$ with $D_{1}=\operatorname{dom}\left(\rho_{1}\right) \subseteq D$ applicable to $u$. By Lemma 32, $\mathcal{E}_{\rho_{1}(u)} \approx \operatorname{flip}_{D_{1}}\left(\mathcal{E}_{u}\right)$ and $D_{1}=\operatorname{odom}\left(\rho_{1}\right)=\operatorname{dom}\left(\rho_{1}\right)$. Thus, flip $_{D \backslash D_{1}}\left(M_{\rho_{1}(u)}\right) \in \theta_{\rho_{1}(u)}$. Now by iteration, there is a reduced sequence $\varphi$ of dual string rules applicable to $u$ such that $\operatorname{odom}(\varphi)=\operatorname{dom}(\varphi)=D$. 
It follows from Lemma 32 and Lemma 33 that reduced sequences of dual string rules are a normal form of sequences of dual string rules. Indeed, by Lemma 32 , if $\varphi$ is a sequence of dual string rules applicable to a legal string $u$ with $D=$ $\operatorname{odom}(\varphi)$, then $\operatorname{flip}_{D}\left(M_{u}\right) \in \theta_{u}$. By Lemma 33, there is a reduced sequence $\varphi^{\prime}$ of dual string rules applicable to $u$ such that $\operatorname{dom}\left(\varphi^{\prime}\right)=\operatorname{odom}\left(\varphi^{\prime}\right)=D$. By the paragraph below Lemma 32 , we have $\varphi(u) \approx \varphi^{\prime}(u)$.

We are now ready to prove the second (and final) main result of this paper. It shows that $\mathcal{R}^{-1}\left(\mathcal{R}_{u}\right)$ (modulo graph isomorphism) is the 'orbit' of $u$ under the dual string rules. That is, the legal strings obtained from $u$ by applying dual string rules are exactly those legal strings to have the same reduction graph as $u$ (up to isomorphism).

\section{Theorem 34}

Let $u$ and $v$ be legal strings. Then $u \approx_{d} v$ iff $\mathcal{R}_{u} \approx \mathcal{R}_{v}$.

\section{Proof}

The forward implication follows directly from Lemma 32 . We now prove the reverse implication. Let $\mathcal{R}_{u} \approx \mathcal{R}_{v}$. By Corollary [1], there is a $E \in \theta_{u}$ such that $\mathcal{E}_{v} \approx B\left(E_{1}, E_{2}, E\right)$ with $\mathcal{R}_{u}=B\left(E_{1}, E_{2}\right)$. By Theorem [15, $E=\operatorname{flip}_{D}\left(M_{u}\right)$ for some $D \subseteq \operatorname{dom}(u)$. Since flip ${ }_{D}\left(M_{u}\right) \in \theta_{u}$, by Lemma 33, there is a reduced sequence $\varphi$ of dual string rules applicable to $u \operatorname{such}$ that $\operatorname{dom}(\varphi)=D$. Now by Lemma 32, $\mathcal{E}_{\varphi(u)} \approx \operatorname{flip}_{D}\left(\mathcal{E}_{u}\right) \approx \mathcal{E}_{v}$, and therefore, by Theorem [10, $\varphi(u) \approx v$.

\section{Discussion}

This paper characterizes, having $\mathcal{R}$ as the function which assigns to each legal string $u$ its reduction graph $\mathcal{R}_{u}$, the range of $\mathcal{R}$ (Theorem 24) and each fiber $\mathcal{R}^{-1}\left(\mathcal{R}_{u}\right)$ modulo graph isomorphism (Theorem 34).

The first characterization corresponds to a computationally efficient algorithm that determines whether or not a graph $G$ is isomorphic to a reduction graph. Moreover, if this is the case, then the algorithm given below Theorem 24 allows for an efficient determination of a legal string $u$ such that $G \approx \mathcal{R}_{u}$. The first characterization relies on the notion of merge-legal edges and its flip operation introduced in this paper. In particular, the connected components in the subgraph induced by the reality edges and the merge-legal edges and the flip operation turns out to be relevant in this context.

The second characterization determines, given $u$, the whole set $\mathcal{R}^{-1}\left(\mathcal{R}_{u}\right)$ modulo graph isomorphism. From a biological point of view, the fibers characterize which micronuclear genes obtain the same macronuclear structure. It turns out that $\mathcal{R}^{-1}\left(\mathcal{R}_{u}\right)$ is the orbit of $u$ under the dual string rules. Surprisingly, these two types of string rewriting rules are very similar to the string positive rules and the string double rules that are used to define the model. Moreover, each two legal strings $u$ and $v$ in such a fiber can be transformed into each other by a sequence $\varphi$ of string rewriting rules without using a pointer more than once. Therefore, the number of string rewriting rules in $\varphi$ can be bounded by the size of the domain of $u$ (and $v$ ).

The reduction graph of a legal string $u$ in a certain sense retains all information regarding applicability of string negative rules in each successful reduction of 
$u$, while discarding almost all other information regarding the rules applied in successful reductions, see 3 . Therefore, the fiber in a sense characterizes all legal strings that have the same properties regarding the application of string negative rules. In biological terms, this may allow for a way to determine whether or not the strategies regarding the string negative rule are different among the different kinds of (genes in) ciliates.

\section{References}

[1] A. Bergeron, J. Mixtacki, and J. Stoye. On sorting by translocations. In S. Miyano et al., editors, RECOMB, volume 3500 of $L N C S$, pages $615-629$. Springer, 2005.

[2] R. Brijder, H.J. Hoogeboom, and M. Muskulus. Applicability of loop recombination in ciliates using the breakpoint graph. In M.R. Berthold et al., editors, CompLife '06, volume 4216 of LNCS, pages 97-106. Springer, 2006.

[3] R. Brijder, H.J. Hoogeboom, and M. Muskulus. Strategies of loop recombination in ciliates. LIACS Technical Report 2006-01, [arXiv:cs.LO/0601135], 2006.

[4] R. Brijder, H.J. Hoogeboom, and G. Rozenberg. The breakpoint graph in ciliates. In M.R. Berthold et al., editors, CompLife '05, volume 3695 of LNCS, pages 128-139. Springer, 2005.

[5] R. Brijder, H.J. Hoogeboom, and G. Rozenberg. Reducibility of gene patterns in ciliates using the breakpoint graph. Theor. Comput. Sci., 356:26$45,2006$.

[6] A.R.O. Cavalcanti, T.H. Clarke, and L.F. Landweber. MDS_IES_DB: a database of macronuclear and micronuclear genes in spirotrichous ciliates. Nucleic Acids Res., 33:D396-D398, 2005.

[7] A. Ehrenfeucht, T. Harju, I. Petre, D.M. Prescott, and G. Rozenberg. Computation in Living Cells - Gene Assembly in Ciliates. Springer Verlag, 2004.

[8] S. Hannenhalli and P.A. Pevzner. Transforming cabbage into turnip: Polynomial algorithm for sorting signed permutations by reversals. J. ACM, 46(1):1-27, 1999 .

[9] P.A. Pevzner. Computational Molecular Biology: An Algorithmic Approach. MIT Press, 2000.

[10] J.C. Setubal and J. Meidanis. Introduction to Computional Molecular Biology. PWS Publishing Company, 1997. 